\title{
Новый механизм поляризации полупроводника на интерфейсе с органическим диэлектриком
}

\author{
(C) А.М. Яфяясов ${ }^{1}$, В.Б. Божевольнов ${ }^{1}$, Е.И. Рюмцев ${ }^{1}$, А.П. Ковшик ${ }^{1}$, В.Ю. Михайловский ${ }^{2}$ \\ ${ }^{1}$ Санкт-Петербургский государственный университет, \\ 199034 Санкт-Петербург, Россия \\ ${ }^{2}$ Междисциплинарный ресурсный центр „Нанотехнологии“ СПбГУ, \\ 198504 Санкт-Петербург, Петродворец, Россия \\ E-mail: yafyasov@gmail.com
}

(Получена 19 мая 2016 г. Принята к печати 31 мая 2016 г.)

Создана система полупроводник-органический диэлектрик с пространственно распределенным зарядом, обладающая уникально низкой плотностью быстрых поверхностных состояний $\left(N_{s s}\right)$ на интерфейсе. На примере $n$-Ge реализована система с $N_{s s} \approx 5 \cdot 10^{10} \mathrm{~cm}^{-2}$ и проведено исследование физических характеристик такой системы с жидкостным и металлическим полевыми электродами. В системе с органическим диэлектриком впервые реализован диапазон изменения поверхностного потенциала от обогащения области пространственного заряда полупроводника основными носителями заряда до состояния инверсии без изменения механизма взаимодействия адсорбированного слоя с поверхностью полупроводника. Обнаружен эффект усиления поляризации области пространственного заряда полупроводника вследствие изменения пространственной структуры подвижного заряда в органическом диэлектрическом слое. На основе разработанной системы открываются технологические возможности для формирования новой генерации электронных приборов на органических пленочных структурах и экспериментального моделирования электронных свойств биологических мембран.

DOI: $10.21883 /$ FTP.2017.02.44105.8331

Изучение процессов в сложных [1] зарядовых системах, возникающих на межфазной границе полупроводника в результате адсорбции [2], является актуальной задачей физики наносистем. В этой связи особый интерес направлен на изучение свойств систем со слоем адсорбированных органических молекул [3], что обусловлено ключевой ролью сложных зарядовых процессов в этих слоях [4] при формировании параметров интерфейса в целом. Результаты этих исследований открывают новые возможности для создания электронных приборов с низкой плотностью быстрых поверхностных состояний (БПС) вблизи середины запрещенной зоны, а также для экспериментального моделирования электрофизических свойств биологических мембран.

В данной работе реализована и изучена система на основе традиционной для исследований в системе полупроводник-электролит $[5,6]$ подложки $n-\mathrm{Ge}$ с ориентацией поверхности (111) и удельным сопротивлением $\rho_{\mathrm{Ge}}=5 \mathrm{OM} \cdot \mathrm{cm}$. Органический диэлектрик (OD) с объемной структурой электрических диполей был подобран таким образом, чтобы обеспечить низкую плотность БПС $\left(N_{s s}<5 \cdot 10^{10} \mathrm{~cm}^{-2}\right)$ на интерфейсе. Низкая плотность БПС на исходной поверхности достигалась контролируемым электрохимическим травлением. Пленка диэлектрика наносилась на поверхность германия методом центрифугирования.

В эксперименте по методике, изложенной в работах $[5,6]$, измерены вольт-амперные (BAX) и вольтфарадные (ВФХ) характеристики интерфейсов $\mathrm{Ge}$ и $\mathrm{Ge}-\mathrm{OD}$ в системе с жидкостным и металлическим полевыми электродами при циклическом изменении элек- тродного потенциала. BAX системы Ge-OD-электролит в сравнении с системой $\mathrm{Ge}-$ электролит демонстрирует уменьшение более чем на порядок тока во всем диапазоне потенциалов поляризации, что свидетельствует о сплошности сформированного OD-слоя, а уменьшение катодного тока $\left(V_{s}>0\right.$, где $V_{s}-$ поверхностный потенциал полупроводника) дополнительно указывает на низкую проницаемость интерфейса с OD-слоем для протонов [5,7].

Для классического интерфейса $n$ - $\mathrm{Ge}-$ электролит $[5,7]$ и его вариаций $[8,9]$ обогащение области пространственного заряда (ОПЗ) свободными носителями наблюдается при гидрированном $(\mathrm{Ge}-\mathrm{H})$, а область инверсии при гидратированном $(\mathrm{Ge}-\mathrm{OH})$ состоянии интерфейса. В противоположность этому интерфейс системы

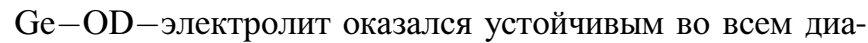
пазоне изменения поверхностного потенциала в ОПЗ германия. При этом концентрация дополнительного встроенного положительного заряда в OD-слое составила: $N_{\mathrm{OD}}=C_{\min } \Delta V / q \approx 2 \cdot 10^{10} \mathrm{~cm}^{-2}$, где $\Delta V$ - относительный сдвиг потенциала минимумов емкости (см. $C_{\min }$ на рис. 1), $q$ - заряд электрона.

Особенности поляризации интерфейса Ge-OD проиллюстрированы на рис. 2, где показаны емкость диэлектрического слоя $\left(C_{\mathrm{OD}}\right)$, вычисленная по ВФХ систем $\mathrm{Ge}$ и $\mathrm{Ge}-\mathrm{OD}-$ электролит, соответствующих анодкатодному направлению изменения потенциала поляризации при их совмещении в точках потенциала плоских зон $\left(V_{s}=V_{f b}=0\right)[5]$ и отношение толщины OD-слоя к его диэлектрической проницаемости $\left(d / \varepsilon_{\mathrm{OD}}\right)$ как функции поляризации OD-слоя. 


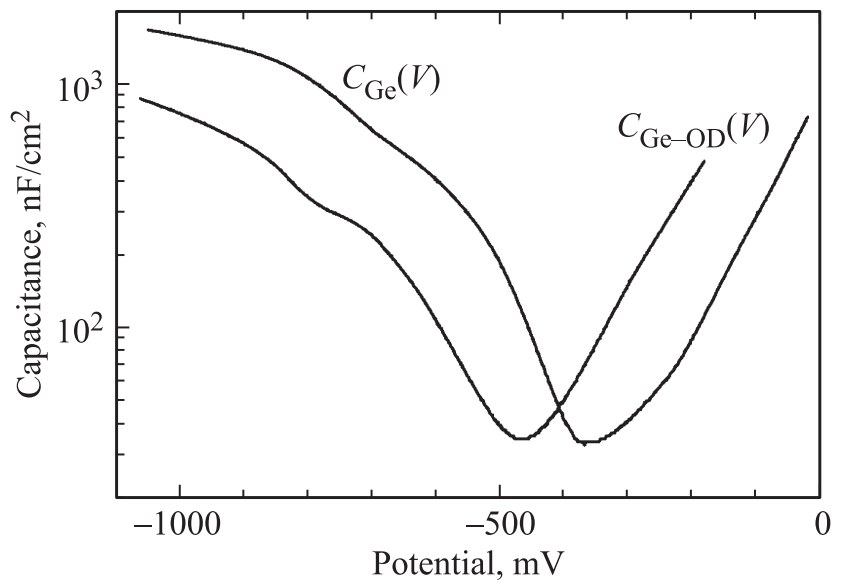

Рис. 1. ВФХ интерфейса $\mathrm{Ge}-$ электролит $\left(C_{\mathrm{Ge}}(V)\right)$ и $\mathrm{Ge}-\mathrm{OD}-$ электролит $\left(C_{\mathrm{Ge}-\mathrm{OD}}(V)\right)$ при изменении потенциала $(V)$ из анода $\left(V_{s}>0\right)$ в катод $\left(V_{s}<0\right), V_{s}$ - поверхностный потенциал полупроводника, $V$ - потенциал электрода поляризации.

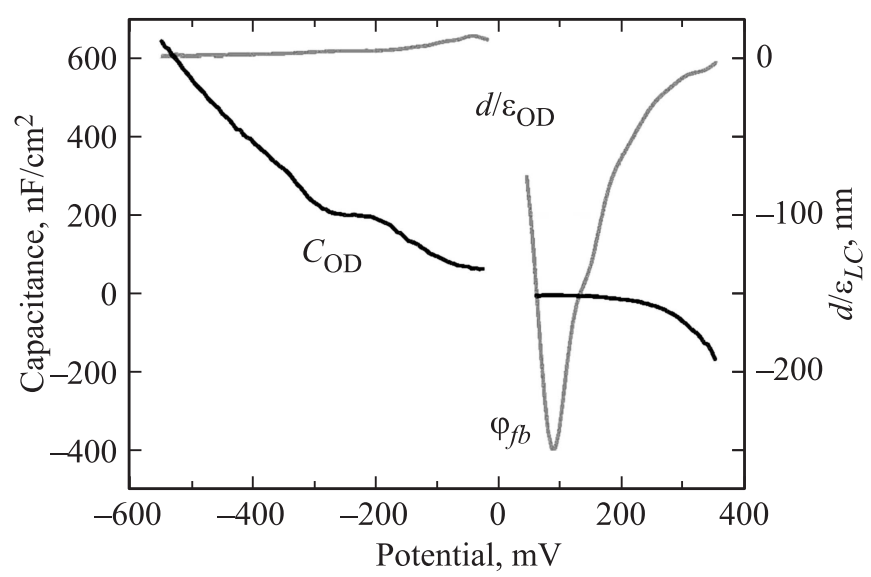

Рис. 2. Емкость диэлектрического слоя $\left(C_{\mathrm{OD}}\right)$, вычисленная по $C_{\mathrm{Ge}}(V)$ и $C_{\mathrm{Ge}-\mathrm{OD}}(V)$ при их совмещении в точках потенциала плоских зон $\left(V_{s}=V_{f b}=0\right)$, и отношение толщины OD-слоя к его диэлектрической проницаемости $\left(d / \varepsilon_{\mathrm{OD}}\right)$ как функции поляризации OD-слоя. Разрыв на приведенных зависимостях в окрестности нулевых значений потенциала связан с методическим ограничением точности вычисления.

Поведение OD-слоя при катодной поляризации подтверждает тот факт, что интерфейс $\mathrm{Ge}-\mathrm{OD}$ сформирован путем взаимодействия положительно заряженных центров OD-слоя с отрицательным зарядом на поверхности полупроводника. При этом отношение $d / \varepsilon_{\mathrm{OD}}$ монотонно меняется от 12 до $1.4 \mathrm{Hм}$.

Анодная поляризация связана с накоплением положительного заряда в ОП3 Ge [5,7]. При этом в интервале потенциалов $0<V<3 k T / q$ (где $k-$ постоянная Больцмана, $T$ - абсолютная температура) наблюдается смена знака дипольного скачка потенциалов в OD-слое (см. рис. 2) без изменения механизма взаимодействия адсорбированного слоя с подложкой. Данное поведение интерфейса могло бы свидетельствовать о перезарядке пика плотности БПС вблизи середины запрещенной зоны $\mathrm{Ge}$, однако наблюдаемая полуширина данного пика менее $4 k T$ делает эту гипотезу маловероятной [6]. Дальнейшее усиление анодной поляризации $(V>3 k T / q)$ приводит к поэтапному (см. „ступенчатая структура“ на $d / \varepsilon_{\mathrm{OD}}$ зависимости с $\Delta V \approx 1.5 k T / q$, см. рис. 2) упорядочению OD-слоя в электрическом поле.

Уникальным свойством системы является сохранение механизма взаимодействия зарядовых центров, обеспечивающих адсорбцию слоя органического диэлектрика на поверхности $\mathrm{Ge}$, независимо от знака заряда в ОПЗ полупроводника. При этом изменение толщины OD-слоя во внешнем электрическом поле приводит к дополнительному симбатному изменению электрического поля в ОПЗ полупроводника и может дать альтернативное „high-k“ диэлектрикам направление создания MOSFET структур. Выяснение механизма усиления поляризации за счет электромеханических процессов в $\mathrm{OD}-$ слое диэлектрика требует проведения дополнительных исследований.

Для выявления роли пространственной подвижности молекул OD-слоя в механизме аномально широкого изменения поверхностного потенциала мы зафиксировали внешнюю поверхность органического диэлектрика металлическим полевым электродом толщиной $\approx 50 \mathrm{Hм}$. Электрод был сформирован распылением мишени $\mathrm{Au} / \mathrm{Pd}$ (30/70) при $10^{-4}$ Па в процессе ее бомбардировки ионами аргона. Ван-дер-ваальсовское взаимодействие металлической матрицы с органическими молекулами воспрепятствовало их пространственному движению.

Пространственная фиксация внешней поверхности OD-слоя привела к исчезновению области инверсии на ВФХ (см. рис. 3) и появлению тока из ОПЗ Ge на металлический электрод при катодной поляризации. Зависимость Шоттки-Мотта для МДП-структуры (рис. 3)

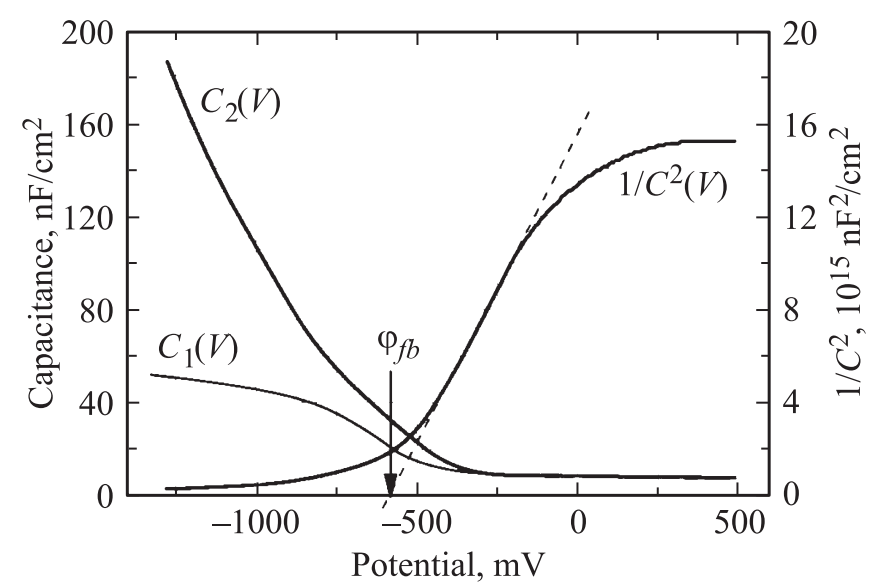

Рис. 3. Вольт-фарадные характеристики $\left(C_{1,2}(V)\right)$ и зависимость Шоттки-Мотта $C^{-2}(V)$ для интерфейса $\mathrm{Ge}-\mathrm{OD}-$ металлический полевой электрод. По мере увеличения силы прижима контакта к напыленному полевому электроду емкость $\left(\right.$ см. $\left.C_{1}(V)\right)$ растет и стремится к насыщению (см. $\left.C_{2}(V)\right)$. 
дает уровень легирования, который согласуется с паспортным значением для подложки $\mathrm{Ge}\left(3.2 \cdot 10^{14} \mathrm{~cm}^{-3}\right)$. Плотность БПС структуры оказалась менее $10^{11} \mathrm{~cm}^{-2}$, что в 2 раза превышает плотность БПС в системе с не зафиксированной внешней поверхностью OD-слоя.

BAX для данной структуры обнаружила линейную зависимость тока от приложенного напряжения с дифференциальным сопротивлением $\approx 150$ Ом $/ \mathrm{cm}^{2}$. Связывая данную особенность с проводимостью пленки, можно оценить подвижность носителей заряда в системе. С точностью до величины $d / \varepsilon$ при концентрации носителей заряда $n=N_{\mathrm{OD}}^{3 / 2} \approx 10^{15} \mathrm{~cm}^{-3}$ получаем значение для подвижности $\left.\approx 2 \cdot 10^{-4} \mathrm{~cm}^{2} / \mathrm{B} \cdot \mathrm{c}\right)$. Данная величина подвижности соответствует либо переносу катионов, либо прыжковой электронной проводимости. По нашему мнению, проводимость OD-слоя связана с конформационной подвижностью элементов органического диэлектрика, обладающего пространственно распределенным зарядом.

Таким образом создана система полупроводник-органический диэлектрик с пространственно распределенным зарядом, обладающая уникально низкой плотностью быстрых поверхностных состояний $\left(N_{s s}\right)$ на интерфейсе. На примере $n$-Ge реализована система с $N_{s s} \approx 5 \cdot 10^{10} \mathrm{~cm}^{-2}$ и проведено исследование физических характеристик такой системы с жидкостным и металлическим полевыми электродами. В системе с органическим диэлектриком впервые реализован диапазон изменения поверхностного потенциала от обагощения области пространственного заряда полупроводника основными носителями до состояния инверсии без изменения механизма взаимодействия адсорбированного слоя с поверхностью полупроводника. Обнаружен эффект усиления поляризации области пространственного заряда полупроводника вследствие изменения пространственной структуры подвижного заряда в органическом диэлектрическом слое. Продемонстрирована эффективность метода эффекта поля с жидкостным полевым электродом для in situ контроля процессов на сложном интерфейсе полупроводник-органический диэлектрик. Это открывает новые возможности как для исследования свойств таких интерфейсов и разработки электронных приборов нового поколения на органических пленочных структурах, так и для экспериментального моделирования электронных свойств биосистем.

Работа выполнена при частичной финансовой поддержке СПбГУ гранта № 11.37.161.2014.

\section{Список литературы}

[1] И.Р. Пригожин, Г. Николис. Самоорганизация в неравновесных системах. От диссипативных структур к упорядоченности через флуктуации (М., Мир, 1979) c. 512 .

[2] Ф.Ф. Волькенштейн. УФН, 9, 275 (1966).
[3] Handbook of Organic Electronics and Photonics (3-Volume Set), ed. by Nalwa H.S. (American Scientific Publishers, 2008).

[4] Я. Бартонь, А.А. Кальнин. ЖТФ, 68, 125 (1998).

[5] P.P. Konorov, A.M. Yafyasov, V.B. Bogevolnov. Field Effect in Semiconductor-Electrolyte Interfaces (Princeton University Press, 2006).

[6] П.П. Коноров, А.М. Яфясов. Физика поверхности полупроводниковых электродов (СПбГУ, Изд-во СПбГУ, 2003) c. 530 .

[7] W.H. Brattain, P.J. Boddy. J. Electrochem. Soc., 109, 572 (1962).

[8] А.М. Яфясов, В.М. Бакулев, П.П. Коноров, В.Б. Божевольнов. ФТП, 45, 1617 (2010).

[9] В.Б. Божевольнов, А.М. Яфясов. Вестн. СПбГУ. Сер. 4, вып. 2, 116 (2004).

Редактор А.Н. Смирнов

\section{A new mechanism for the polarization of the semiconductor at the interface with the organic dielectric}

\author{
A.M. Yafyasov ${ }^{1}$, V.B. Bogevolnov ${ }^{1}$, E.I. Ryumtsev ${ }^{1}$, \\ A.P. Kovshik ${ }^{1}$, V.Yu. Mikhailovski ${ }^{2}$ \\ ${ }^{1}$ Saint-Petersburg State University, \\ 199034 St. Petersburg, Russia \\ ${ }^{2}$ Interdisciplinary Resource Center \\ "Nanotechnology" of SPbU, \\ 198504 St. Petersburg, Russia
}

\begin{abstract}
The unique low-density fast surface states $\left(N_{S S}\right)$ on the semiconductor - an organic insulator interface was able to realize through choose the structure of the spatial charge distribution in organic molecules. The physical characteristics of the interface was measured by field effect with liquid and metal electrodes. The germanium interface for both electrodes have $N_{S S} \approx 5 \cdot 10^{10} \mathrm{~cm}^{-2}$. In this system, first implemented the surface potential change from the inversion to accumulation of charge carriers at the semiconductor surface without changing the mechanism of interaction between the organic layer and the semiconductor surface. The effect of increasing polarization spacecharge region of the semiconductor occurs due to changes in the spatial structure of the mobile charge in the organic dielectric layer. This interface gives a technological opportunities for the formation of a new generation of electronic devices based on organic films and for experimental modeling of electronic properties of biological membranes.
\end{abstract}

